\title{
An Emission-Aware Day-Ahead Power Scheduling System for I nternet of Energy
}

\author{
Chenn-Jung Huang ${ }^{1,2 *}$, Kai-Wen $\mathrm{Hu}^{2}$, An-Feng Liu ${ }^{1}$, Liang-Chun Chen ${ }^{3}$, and Chih-Ting Chen ${ }^{1}$ \\ ${ }^{1}$ Department of Computer Science \& Information Engineering, National Dong Hwa University \\ ${ }^{2}$ Department of Electrical Engineering, National Dong Hwa University \\ ${ }^{3}$ Department of Management, Fo Guang University, Yilan, Taiwan. \\ *Corresponding author : Chenn-Jung Huang
}

Received August 25, 2018; revised November 23, 2018; accepted April 16, 2019;

published October 31, 2019

\begin{abstract}
As a subset of the Internet of Things, the Internet of Energy (IoE) is expected to tackle the problems faced by the current smart grid framework. Notably, the conventional day-ahead power scheduling of the smart grid should be redesigned in the IoE architecture to take into consideration the intermittence of scattered renewable generations, large amounts of power consumption data, and the uncertainty of the arrival time of electric vehicles (EVs). Accordingly, a day-ahead power scheduling system for the future IoE is proposed in this research to maximize the usage of distributed renewables and reduce carbon emission caused by the traditional power generation. Meanwhile, flexible charging mechanism of EVs is employed to provide preferred charging options for moving EVs and flatten the load profile simultaneously. The simulation results revealed that the proposed power scheduling mechanism not only achieves emission reduction and balances power load and supply effectively, but also fits each individual EV user’s preference.
\end{abstract}

Keywords: power scheduling, electric vehicle charging, emission reduction, soft computing, Internet of Energy 


\section{Introduction}

In the past decade, numerous research efforts have been devoted to the issues of emission reduction and efficient usage of the penetrated renewables. Recently, a newly introduced energy network concept, called the Internet of Energy (IoE), is adopted by researchers to tackle the power management problems caused by the surging growth of dispersed renewables, charging/discharging of electric vehicles (EVs) and battery storage, and smart meters [1]. Similar to the approach taken in the Internet, energy routers [2] is employed in the IoE framework to interconnect dispersed power generations and assist in energy sharing of the whole power market on a large scale [3].

On the basis of present levels of information integration, the IoE coordinates the owners of dispersed power generations to sell the power to others facing power shortage during peak load periods [4]. A suitable day-ahead power scheduling scheme that achieves load peak reduction is demanded to timely collect and analyze the increasingly data collected from the dispersed power generations, smart metering infrastructure, and charging/discharging of EVs and battery storage facilities in order to assist in the power market operator optimizing short-term power usage to prevent the power crisis from surging demand during peak loads and deteriorating greenhouse effect.

In the recent literature, several researchers proposed algorithms to tackle the short-term power problem for the future IoE. Hou et al. formulated the power exchange and storage problem for IoEs and presented heuristics to solve the optimization problem by transforming the NP-hard optimization into classic knapsack problem [4]. Zhang et al. took into consideration the intermittence of renewable generations and proposed a distributed consensus-ADMM power management algorithm [5]. The power management problem for the IoE was formulated as a three-stage Stackelberg game in the work of Zhou et al. [6]. The microgrid, the power utility company, and the consumers act as the players in Stackelberg game, and all players' profits and the power market reliability were effectively achieved. Lin et al. optimized the IoE operation by allowing prosumers to employ battery storage utilities to charge and discharge the electricity and shifted the peak load demand effectively [7]. Du et al. presented a hierarchical power management scheme for the IoE to exchange tremendous amounts of power and information for renewable-grid deployment on a large scale [8]. Dou et al. took power network security into consideration and developed four types of differential hybrid Petri-net control mechanism, in which the power generated by 
distributed renewables were fully utilized to meet peak electricity load [9].

The integration of EVs and battery storage utilities with microgrids have been studied by researchers in recent years as well. Tang and Zhang tackled the EV charging problem via a predictive dynamic programming control model [10]. Renewable was installed at each charging station, and the power can be traded among the charging stations and microgrids. Markov processes were applied to characterize the intermittence of the renewables, the volatile charging demand of the EVs, and the real-time variation of the electricity price. Wang et al. formulated EV charging problem into two-phase processes [11]. An offline power scheduling scheme was initiated first to determine the amount of power supply during each hour day ahead. A real-time EV charging algorithm was then executed to derive the dynamic EV charging rate. Kang et al. adopted the real-time electricity price, charging location, and charging priority as input parameters to an EV battery swapping service. Minimization of power loss, charging cost, and peak load demand were achieved in their work [12]. Ghasemi et al. integrated EV batteries and battery storage facilities with wind farms to smooth the curve of power supply and demand for the electricity market. EV charging were performed during off-peak periods in order to cut down the EV charging cost [13]. Assuming that a prosumer owns solar and wind power generation, EV, and battery storage facility, Lin et al. established a mixed-integer linear programming energy trading platform among prosumers to minimize their electricity costs [14]. A hierarchical game mechanism was adopted by Tan and Wang to navigate EVs to suitable charging stations under the scenario of the transportation and power network [15]. Their simulation results showed that the Nash equilibrium was found to increase the revenues of charging stations and enhance the stability of power market.

From the recent literature cited above, it can be seen that the researchers seldom jointly considered load profile, renewable generation, EV charging, battery storage facility of an individual prosumer, and cooperative operations of microgrids together into the design of the day-ahead power scheduling for the IoE framework. Meanwhile, to the best of our knowledge, little research work integrates charging demand of moving EVs with the day-ahead power scheduling of the future IoE. Thus, a day-ahead power scheduling mechanism is proposed in this work to address the technical management issues raised by the extensive deployment of intermittent renewables, advanced metering infrastructure, and charging need of moving EVs. Regional energy routers are deployed at each microgrid to collect and analyze the day-ahead data of power supply and demand for each household hourly. Then, power scheduling is initiated to fully utilize the renewable power generations, 
reduce the electricity bills of prosumers and mitigate carbon emission caused by the traditional power generation. Notably, flexible charging service is proposed here to assist moving EVs in selecting suitable charging options in order to satisfy the charging demand for the EV owners with various preferences and balance the charging loading for all charging points. Furthermore, flexible charging service is integrated with the power scheduling to alleviate the peak electricity load for the whole IoE service territory. The rest of this article is organized as follows. In Section 2, we present the day-ahead power scheduling algorithm. The simulation results are provided and analyzed in Sections 3. Finally, the conclusion is given in Section 4.

\section{Architecture of the day-ahead power scheduling system for the IoE}

The service territory of the IoE in this work is composed of multiple geographical areas, and the microgrids located within each geographical area is managed by the same regional energy router. A hierarchical framework is constructed and greater numbers of hierarchical levels are required for broad geographical areas or highly populated metropolitan cities. The residences/buildings within the service territory of each microgrid can be equipped with solar or wind power generation systems and battery storage facilities, and the surplus solar or wind power can be stored in the battery storage facility at each residence/building. Once the battery storage facility is fully charged, the surplus power can be traded and be kept at the battery storage facility owned by the microgrid upstream. The microgrid can utilize the stored power to satisfy the electricity load demand of public utilities within the territory managed by the microgrid. Notably, via the information exchange among regional energy routers, the surplus power can also be traded to the moving EVs that are expected to reach the microgrid that generates power or the prosumers/microgrids that lack of power during peak load periods.

Along with surging growth of EV market in recent years, the provision of charging facilities turns out to be one major concern for potential EV buyers, besides the purchase price of the EV. In the literature, most research work has been devoted to charge/discharge management of parked EVs [16]. Recently, several researchers investigated charging problems for moving EVs. Apart from the conventional plug-in charging station, different charging options, such as EV battery switching [17] and on-road wireless charging [18]-[20], were presented to provide more selections for EV charging methods. However, the integration of the EV user's charging preference with the route planning should be considered to fit each individual EV user's need. 
As shown on the bottom right of Fig. 1, each EV is set up to run its trip planning and charging checking module. In order to update the location of the EV in real-time, the EV estimates the battery status of the EV on the way to the destination during each fixed short-time interval. Since the development of on-board vehicle route planning systems is already mature [21][22], this work applies any available trip planning software package on the market to estimate the time required to reach the destination. The proposed trip planning and charging checking module then checks the battery status of the moving EV. In case electricity shortage occurs midway in the trip, this module will locate the most suitable charging point on the way to the destination that fits the EV user's preference.

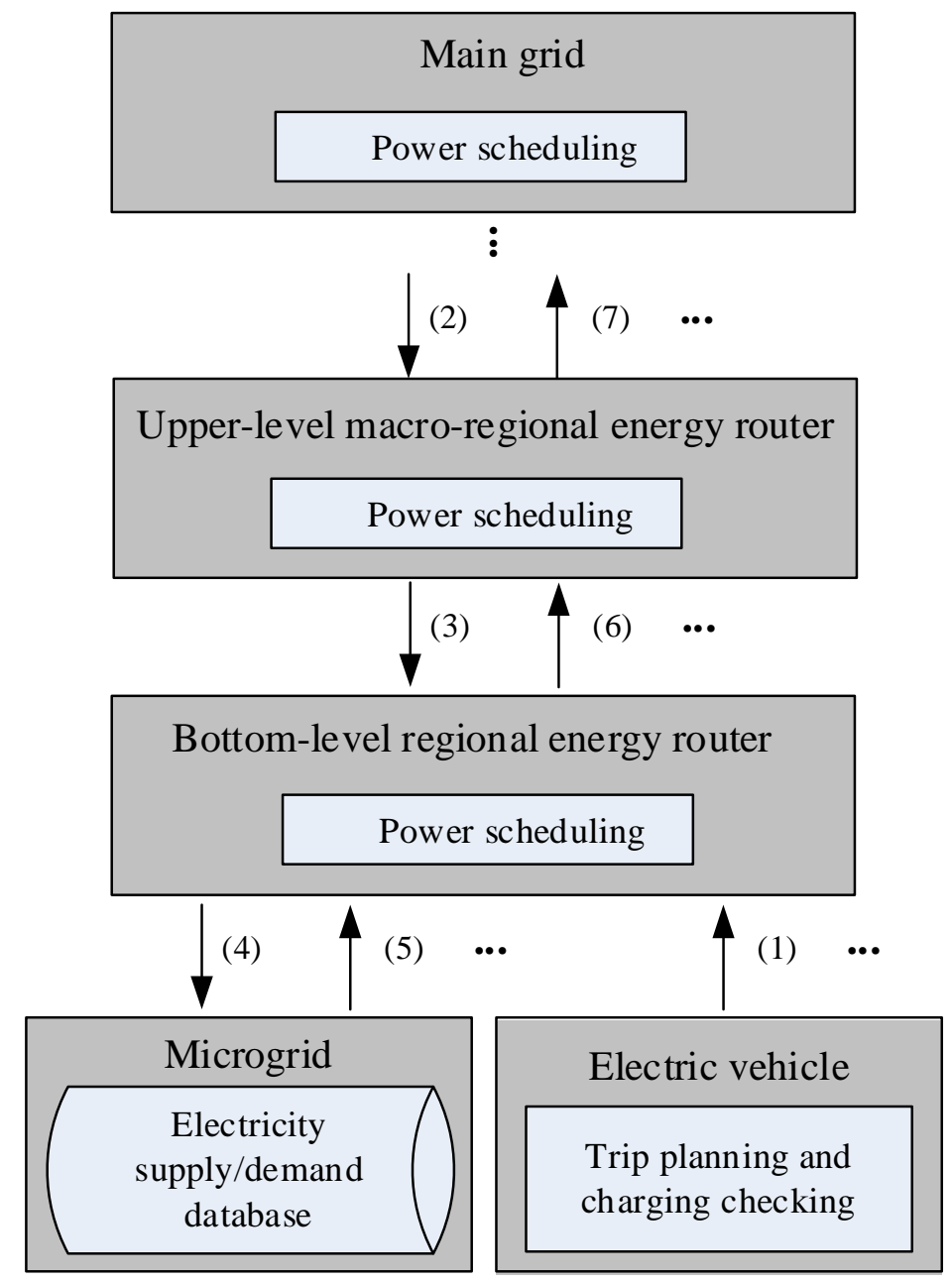

Fig. 1. Architecture the proposed power scheduling algorithm

There are four options of charging point selection for the EV user, including slow charging station, fast charging stations, battery switching station, and on-road wireless 
charging. For example, if the EV user wishes to reach the destination as soon as possible, the trip planning and charging module will determine the charging option for a moving EV that takes the shortest time to reach and wait at the charging point. On the contrary, the EV user is suggested to select the charging point with the lowest charging cost if no specific charging preference is designated. In this work, all charging options on the way to the destination is divided into two groups, one supporting fast charging or on-road wireless charging, and the other offering low-cost/slow charging service. That is, the charging points offering low-cost/slow charging services are placed into the candidate list if the major concern of the EV owner is the charging price, whereas other charging options that provide fast charging or on-road wireless charging will be recorded in the cache if the EV owner expects to recharge the battery as fast as possible.

After the EV updates all candidate charging points that suit the preference of the EV in the cache, fuzzy logic technique is applied to determine the most suitable charging option from all candidate charging points. the fuzzy inference system is employed in this work owning to its effectiveness in the power management for EVs in the recent literature [23][24]. After the charging option for the EV is determined, the EV then notifies the regional energy router(s) at which the charging point(s) are located, and provide the regional router all charging related information, including the arrival time at the designated charging point(s), desired minimal state of charge (SoC) of the EV battery, the day-ahead hourly SoC for the EV battery, and.

As illustrated in Fig. 1, the operator of the IoE initiates the power scheduling algorithm at the main grid and forwards the power scheduling requests to the regional energy routers at all levels downstream. Accordingly, the power trading among the microgrids can be carried out by the bottom-level regional energy routers. Notably, if a microgrid is notified by a moving EV that it needs to charge its battery at a specific charging point located within the service territory of the microgrid, the estimated arrival time of the EV, the battery status after the EV arrives at the microgrid, and the expected amount of charged electricity will be recorded at the cache of the bottom-level regional energy router upstream for future scheduling reference.

After scheduling, status reports of hourly power supply and demand is collected by the regional energy router at each level and the overall aggregate power status data is sent to the main grid for review of the updated status of day-ahead power operation. Notably, the computational complexity is considerably reduced in the presented hierarchical framework 
when compared with the traditional centralized control mechanism. The detailed description of each module as depicted in Fig. 1 is given below.

\section{1. scheduling for regional energy router at upper level}

Step 1: The regional energy router at the current upper level requests the regional energy router(s) downstream to schedule the hourly usage of electricity and coordinate the power trading with other regional energy routers at the same level, if necessary. As mentioned above, the renewables are deployed at the prosumer level, and the battery storage facilities can be installed by the prosumers or microgrids in this work. As a result, the regional routers at all levels deal with information exchange and power trading, whereas the actual power generation and consumption occurs at the microgrid and prosumer levels.

Step 2: If the main grid level is not reached yet, go to Step 3. Otherwise, this work makes an effort to minimize the dependency on the traditional power generation at the main grid to reduce carbon emission:

$\operatorname{Min} \sum_{t=1}^{24} T P_{t}$

$\operatorname{Min} \sum_{j} \sum_{t=1}^{24}\left|C P_{D L G_{j}, t}+S L B_{D L G_{j}, t}\right|$

subject to

$T P_{t}+\sum_{j}\left(C P_{D L G_{j}, t}+S L B_{D L G_{j}, t}\right) \geq 0$

$\sum_{t=1}^{24}\left(C P_{D L G_{j}, t}+S L B_{D L G_{j}, t}\right)=\sum_{t=1}^{24} S L B_{D L G_{j}, t}$

where $T P_{t}$ represents the required traditional power generation at time $t$. Notably, $T P_{t}$ is larger than or equal to zero. $S L B_{D L G_{j}, t}$ denotes the status report of hourly power supply and demand submitted by the $j$ th regional energy router downstream, and $C P_{D L G_{j}, t}$ stands for the power traded by the $j$ th regional energy router downstream at time $t$. Notably, the term $\left(C P_{D L G_{j}, t}+S L B_{D L G_{j}, t}\right)$ represents the up-to-date hourly power supply and demand status of the $j$ th regional energy router downstream at time $t$ after this step is executed. Step 4 is executed next from here. 
Step 3: The current regional energy router transforms the flattening of the hourly load profile and the power supply and demand curve within its service territory into the following optimization problem:

$$
\begin{aligned}
& \operatorname{Max} \sum_{i} \sum_{t=1}^{24} R S P C_{D L G_{j}} \\
& \operatorname{Min} \sum_{j} \sum_{t=1}^{24}\left|C P_{D L G_{j}, t}+S L B_{D L G_{j}, t}\right|
\end{aligned}
$$

subject to:

$$
\begin{aligned}
& \sum_{t=1}^{24}\left(C P_{D L G_{j}, t}+S L B_{D L G_{j}, t}\right)=\sum_{t=1}^{24} S L B_{D L G_{j}, t} \\
& R S P C_{D L G_{j}}= \begin{cases}1 & C P_{D L G_{j}, t}+S L B_{D L G_{j}, t} \geq 0 \\
0 & C P_{D L G_{j}, t}+S L B_{D L G_{j}, t}<0\end{cases}
\end{aligned}
$$

Step 4: If the main grid level is not reached, submit the status report of hourly power supply and demand to the regional energy router upstream as follows,

$S L B_{U L G_{u}, t}=\sum_{j}\left(C P_{D L G_{j}, t}+S L B_{D L G_{j}, t}\right)$

Otherwise, the status reports of hourly power supply and demand of the whole IoE service territory are kept at the cache of the main grid, and the hourly traditional power generations $T P_{t}$ is adjusted accordingly.

\subsection{Power scheduling for the regional energy router at the bottom level}

Step 1: Request the microgrids downstream to predict hourly electricity load, renewable power generation, and the SoCs of EV batteries and battery storage facilities.

Step 2: Collect the status of hourly power supply and demand reported by the ith microgrid downstream and calculate the difference between hourly power supply and the non-schedulable demand within the service territory of the regional energy router as follows:

$S L D_{M G_{i}, t}=R E_{M G_{i}, t}-\sum_{m}\left(\sum_{r} L_{M G_{i}, N s h t U_{m, r}, t}+\sum_{s}\left(1-\varsigma_{M G_{i}, U_{m}, t}\right) \cdot L_{M G_{i}, \operatorname{sht} U_{m, s}, t}\right)-\sum_{l} L_{M G_{i}, P S L_{l}, t}$

Description of parameters: 
(i) $R E_{M G_{i}, t}$ represents the total power supply provided by the ith microgrid at time $t$, whereas $L_{M G_{i}, P S L_{l}, t}$ denotes the $l$ th power load of the public service required at the ith microgrid, whereas $L_{M G_{i}, s h t U_{m, s}, t}$ and $L_{M G_{i}, N s h t U_{m, r}, t}$, stand for the sth schedulable and the $r$ th non-schedulable electricity load of the $m$ th prosumer, respectively.

(ii) In this work, the prosumers who keep good historical record in adherence to the former scheduling operations of the IoE can be offered with certain electricity purchase price discount from power trading market in case the prosumers face power shortage during peak load periods. Based on the historical record, each prosumer is judged whether she/he is qualified to get involved in the appliance scheduling operation. If a system variable $\varsigma_{M G_{i}, U_{m}, t}$ is set to one, the schedulable load of the $m$ th prosumer is allowed to participate in the hourly scheduling operation. $\varsigma_{M G_{i}, U_{m}, t}$ can be expressed by:

$$
\varsigma_{M G_{i}, U_{m}, t}= \begin{cases}1 & C_{M G_{i}, U_{m}} \geq C_{M G_{i}, U_{m}}^{t h} \\ 0 & C_{M G_{i}, U_{m}}<C_{M G_{i}, U_{m}}^{t h}\end{cases}
$$

where $C_{M G_{i}, U_{m}}^{t h}$ stands for the threshold of whether the $m$ th prosumer will take part in the hourly scheduling operation. $C_{M G_{i}, U_{m}}$ is derived by:

$C_{M G_{i}, U_{m}}=\left\{\begin{array}{lc}\frac{\left((w-1) C_{M G_{i}, U_{m}}+\varepsilon_{M G_{i}}\right)}{w} & \text { if the } m \text { th prosumer took part in the latest sc } \\ \frac{\left((w-1) C_{M G_{i}, U_{m}}-\varepsilon_{M G_{i}}\right)}{w} & \text { Otherwise }\end{array}\right.$

where $\varepsilon_{M G_{i}}$ and $w$ represent certain fixed constants predetermined by the system operator.

Step 3: There are three optimization objectives at this step, including flattening of the peak load periods, prevention of the hourly power shortage within the service territory of the current regional energy router, and the reduction of the electricity purchase cost for the prosumers who endure power shortage. The formulation of the three-objective optimization problem can be expressed by:

$\operatorname{Max} \sum_{i} \sum_{t=1}^{24} S P C_{M G_{i}, t}$

$\operatorname{Min} \sum_{t=1}^{24}\left|\sum_{i} S L R D_{M G_{i}, t}\right|$ 
$\operatorname{Min} \sum_{t=1}^{24} \sum_{i} c_{M G_{i}, t} \cdot\left(\sum_{m} \sum_{s} \varsigma_{M G_{i}, U_{m}, t} \cdot L_{M G_{i}, s h t U_{m, s}, t}+\sum_{j} \chi_{M G_{i}, E V_{j}, t} \cdot B_{E V_{j}, t}^{c h}+\sum_{k} B_{M G_{i}, M S_{k}, t}^{c h}\right)$

subject to:

$$
\begin{aligned}
& S L R D_{M G_{i}, t}=S L D_{M G_{i}, t}+\sum_{k}\left(B_{M G_{i}, M S_{k}, t}^{d c h}-B_{M G_{i}, M S_{k}, t}^{c h}\right)-\sum_{j} B_{E V_{j}, t}^{c h} \\
& -\sum_{m} \sum_{s} \varsigma_{M G_{i}, U_{m}, t} \cdot L_{M G_{i}, s h t U_{m, s}, t} \\
& S P C_{M G_{i}, t}= \begin{cases}1 & S L R D_{M G_{i}, t} \geq 0 \\
0 & S L R D_{M G_{i}, t}<0\end{cases} \\
& 0 \leq B_{E V_{j}, t}^{c h} \leq \delta_{E V_{j}, t}^{c h} \cdot S o C_{E V_{j}}^{\max } \\
& \operatorname{SoC}_{E V_{j}, t}=S o C_{E V_{j}, t-1}+B_{E V_{j}, t}^{c h} \cdot \eta_{E V_{j}}^{c h} \\
& \operatorname{SoC}_{E V_{j}}^{\min } \leq \operatorname{SoC}_{E V_{j}, t} \leq \operatorname{SoC}_{E V_{j}}^{\max } \\
& \operatorname{SoC}_{E V_{j}, 0}=\operatorname{SoC}_{E V_{j}}^{i n i} \\
& S o C_{M G_{i}, M S_{k}, t}=S o C_{M G_{i}, M S_{k}, t-1}+\left(B_{M G_{i}, M S_{k}, t}^{c h} \cdot \eta_{M G_{i}, M S_{k}}^{c h}-B_{M G_{i}, M S_{k}, t}^{d c h} / \eta_{M G_{i}, M S_{k}}^{d c h}\right) \\
& \operatorname{SoC}_{M G_{i}, M S_{k}}^{\min } \leq \operatorname{SoC}_{M G_{i}, M S_{k}, t} \leq \operatorname{SoC}_{M G_{i}, M S_{k}}^{\max } \\
& \operatorname{SoC}_{M G_{i}, M S_{k}, 0}=\operatorname{SoC}_{M G_{i}, M S_{k}}^{i n i} \\
& 0 \leq \eta_{E V_{j}}^{c h}, \eta_{M G_{i}, M S_{k}}^{c h}, \eta_{M G_{i}, M S_{k}}^{d c h} \leq 1 \\
& 0 \leq B_{M G_{i}, M S_{k}, t}^{d c h} \leq \beta_{M G_{i}, M S_{k}, t} \cdot \delta_{M G_{i}, M S_{k}, t}^{d c h} \cdot \operatorname{SoC}_{M G_{i}, M S_{k}}^{\max } \\
& \beta_{M G_{i}, M S_{k}, t}=\left\{\begin{array}{cc}
1 & \text { if } c_{M G_{i}, t} \cdot B_{M G_{i}, M S_{k}, t}^{d c h} \geq D C_{M G_{i}, M S_{k}, t}^{d c h} \\
0 & \text { otherwise }
\end{array}\right. \\
& \delta_{M G_{i}, M S_{k}, t}^{c h}+\delta_{M G_{i}, M S_{k}, t}^{d c h}=1, \delta_{M G_{i}, M S_{k}, t}^{d c h} \in\{0,1\}, \delta_{M G_{i}, M S_{k}, t}^{c h} \in\{0,1\} \\
& 0 \leq B_{M G_{i}, M S_{k}, t}^{c h} \leq \gamma_{M G_{i}, M S_{k}, t} \cdot \delta_{M G_{i}, M S_{k}, t}^{c h} \cdot \operatorname{SoC}_{M G_{i}, M S_{k}}^{\max } \\
& \gamma_{M G_{i}, M S_{k}, t}=\left\{\begin{array}{cc}
1 & \text { if } c_{M G_{i}, t} \cdot B_{M G_{i}, M S_{k}, t}^{c h} \geq D C_{M G_{i}, M S_{k}, t}^{d c h} \\
0 & \text { otherwise }
\end{array}\right. \\
& c_{M G_{i}, t}=\left\{\begin{array}{cc}
b p_{M G_{i}} & \text { if time } t \text { is during off peak hours } \\
\rho_{M G_{i}} \cdot b p_{M G_{i}} & \text { otherwise }
\end{array}, \rho_{M G_{i}}>1\right. \\
& \chi_{M G_{i}, E V_{j}, t}=\left\{\begin{array}{lc}
1 & \text { if the } j \text { th } \mathrm{EV} \text { arrives at the } i \text { th microgrid at time } t \\
0 & \text { otherwise }
\end{array}\right.
\end{aligned}
$$

Description of parameters:

(i) $B_{E V j}^{c h}, t$ denotes the electricity that the $j$ th $\mathrm{EV}$ gets charged at some specific charging point within the service territory of the regional router at time $t$. $B_{M G_{i}, M S_{k}, t}^{c h}$ and $B_{M G_{i}, M S_{k}, t}^{d c h}$ represent the hourly charging and discharging 
electricity of the $k$ th battery storage facility deployed at the ith microgrid, respectively. $S_{o} C_{E V_{j}, t}$ denote the hourly SoC of the jth EV battery, whereas $S o C_{E V_{j}}^{i n i}$, $S o C_{E V_{j}}^{\max }$ and $S o C_{E V_{j}}^{\min }$ stand for the initial, maximal and minimal electricity stored at the $j$ th EV battery, respectively. $S o C_{M G_{i}, M S_{k}, t}$ denote the hourly SoC for the $k$ th battery storage facility, whereas $S_{o} C_{M G_{i}, M S_{k}}^{i n i}$, $S o C_{M G_{i}, M S_{k}}^{\max }$ and $S_{o C_{M G_{i}, M S_{k}}^{\min }}$ stand for the initial, maximal and minimal electricity stored at the kth battery storage facility, respectively. Notably, this work assumes that the $j$ th EV computes $S_{0} C_{E V_{j}}^{m i n}$ within a short time interval before the initiation of power scheduling of the main grid, and the governing bottom-level regional grid will be notified if the EV needs to get charged at any charging point within the service area of a microgrid at time $t$.

(ii) $\chi_{M G_{i}, E V_{j}, t}$ is set to one if the $j$ th $\mathrm{EV}$ enters the geographical area of the $i$ th microgrid. Otherwise, it is set to zero. The flags $\beta_{M G_{i}, M S_{k}, t}$, and $\gamma_{M G_{i}, M S_{k}, t}$ stands for the thresholds of whether the electricity trading through the discharging/charging of the $k$ th battery storage facility is larger than the degradation cost of the battery. These two flags are set to ones if the electricity trading is larger than the degradation cost.

(iii) $D C_{E V_{j}, t}^{d c h}$ and $D C_{M G_{i}, M s_{k}, t}^{d c h}$ represent the battery degradation cost owing to charging/discharging operation [25]. Binary values of $\delta_{E V_{j}, t}^{c h}$ indicates whether the $j$ th EV is under charging operation mode, whereas $\delta_{M G_{i}, M S_{k}, t}^{c h}$ and $\delta_{M G_{i}, M S_{k}, t}^{d c h}$ indicate whether the $k$ th battery storage facility is charging or discharging, respectively. $\eta_{E V_{j}}^{c h}$ stands for charging efficiency for the $j$ th $\mathrm{EV}$ battery, whereas $\eta_{M G_{i}, M S_{k}}^{c h}$ and $\eta_{M G_{i}, M S_{k}}^{d c h}$ denotes charging and discharging efficiency for the $k$ th battery storage facility, respectively.

(iv) $c_{M G_{i}, t}$ stands for the Time-of-Use (ToU) [26] electricity cost charged for the prosumers at the ith microgrid in real time. $b p_{M G_{i}}$ and $\rho_{M G_{i}}$ stand for the off-peak electricity price and the weighting factor of the peak-hour electricity purchase cost of the prosumers at the ith microgrid, respectively. These two values are updated daily based on the average hourly electricity demand in the past records of the $i$ th microgrid.

Step 4: Submit the status report of hourly power supply and demand to the regional energy router upstream.

\subsection{Trip planning and charging checking for the EV}

Step 1: This work assumes that each EV installs some commercial route planning software package such as the one developed in [21]. The EV computes the travelling time to reach the destination via the route planning software.

Step 2: This work assumes that the average power consumption of the $j$ th EV battery per kilometer is saved at the cache of the $j$ th EV. The $j$ th EV checks its battery status to determine whether its battery runs out of power before reaching the destination. The 
demanded minimal charging power of its battery before arriving at the destination can be derived by:

$$
S o C_{E V_{j}}^{\text {dest }}=\left\{\begin{array}{cc}
S o C_{E V_{j}}^{\min }-S o C_{E V_{j}}^{c u r}+\operatorname{Dist}_{E V_{j}}^{\text {dest }} & \text { if } S o C_{E V_{j}}^{c u r}-A C_{E V_{j}} \cdot \operatorname{Dist}_{E V_{j}}^{\text {dest }}<\operatorname{SoC}_{E V_{j}}^{\text {min }} \\
0 & \text { otherwise }
\end{array}\right.
$$

where $S o C_{E V_{j}}^{\min }$ denotes the minimal electricity threshold that must be preserved at the battery of the $j$ th EV and $S o C_{E V_{j}}^{c u r}$ stands for the current SoC of its battery. $D i s t_{E V_{j}}^{\text {dest }}$ represents the traveling distance between the current position of the EV and the destination, and $A C_{E V_{j}}$ expresses the average EV battery power consumption per kilometer.

Step 3: If the computation at the preceding step indicates no power shortage will occur before the $j$ th EV reaches the destination, exit this module.

Step 4: The EV goes through each charging point on the way to the destination, and add all candidate charging points that suits the preference of the EV in the cache. The charging points offering low-cost/slow charging service are placed into the candidate list if the major concern of the EV owner is the charging price. On the contrary, the charging options that provide fast charging or on-road wireless charging will be recorded at the cache if the EV owner expects the time to reach the destination as short as possible.

Step 5: The EV computes the estimated arrival time at each charging point on the candidate list, and sends a message that includes the estimated arrival time and demanded minimal charging power of the battery to each candidate charging point. Notably, this work assumes the candidate charging points will reply to the querying EV with the hourly charging price and the estimated turnaround time during the arrival of the $\mathrm{EV}$. The definition of turnaround time here is the summation of the waiting and service time for recharging, and all charging services are operated on a first-come-first-served basis.

Step 6: After the EV receives the response from all candidate charging points, a fuzzy charging point selection mechanism is applied to determine the best charging choice that fits the preference of each individual EV user from the candidates. As shown in Fig. 2, there are three input parameters to the fuzzy charging point selection mechanism. They are the hourly electricity cost charged by the charging point, extra travelling distance demanded to get to the charging point, and the turnaround time 
needed for completing charging service at the charging point. As for the output of the fuzzy charging point selection mechanism, it represents a fitness measure of the charging point that serves the EV.

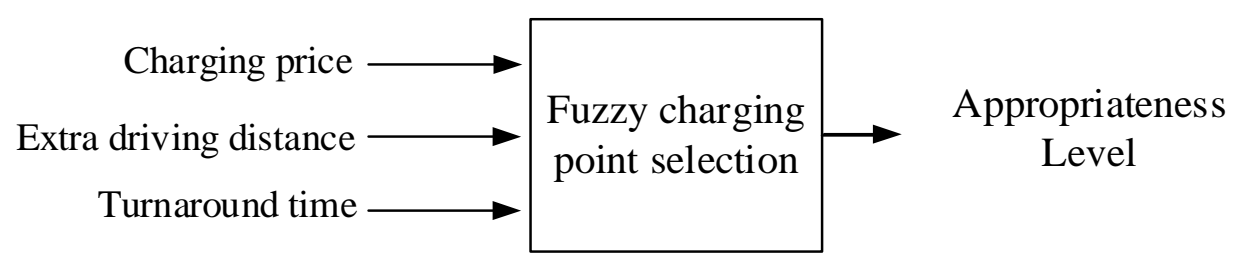

Fig. 2. Illustration of fuzzy charging point selection mechanism.

A set of 27 If-Then rules constructs the fuzzy rule base for the charging point selection procedure. The fuzzy inference engine based on Madamni-type model is applied in this work. An example of the fuzzy If-Then rule is shown in Fig. 3. The three antecedents and the consequent for each rule correspond to the four membership functions from left to right in the figure. Three linguistic terms, "low", "medium" and "high" are used in the membership function for the first antecedent, and three linguistic terms, "short", "medium" and "long” are adopted for the second and third antecedent membership functions, respectively. The fuzzy linguistic variables for the consequent of the inference engine, which is the appropriateness level of the candidate charging point, are "low", “medium” and "high”. The example rule given in Fig. $\mathbf{3}$ can be described as:

IF the hourly electricity cost charged by the charging point is "medium", AND the extra travelling distance to the charging point is "short", AND the turnaround time needed for completing charging service is "medium"

THEN the fitness measure of the charging point that suits the need of the EV is "medium".

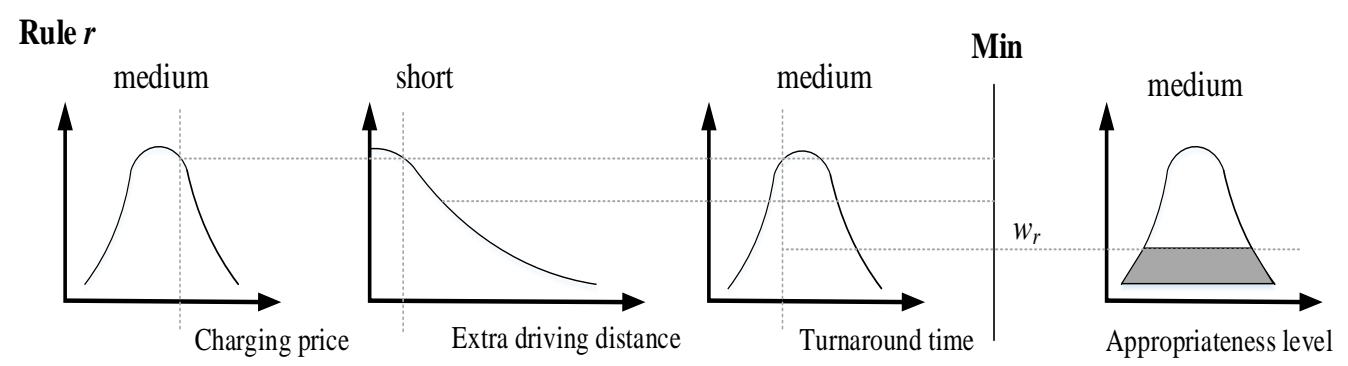

Fig. 3. An example of Mamdani fuzzy inference engine 
Based on the Mamdani-type mode, the crisp output of the fuzzy inference engine is expressed as the weighted average of consequents of all fuzzy If-Then rules:

$$
A L=\frac{\int_{l} \mu_{A}(l) l d l}{\int_{l} \mu_{A}(l) d l},
$$

where $\mu_{A}(l)$ represents the accumulated area of all “AND” antecedents.

Step 7: The EV submits the charging request to the charging point derived at the preceding step and the bottom-level regional energy router that governs the charging point.

\section{Experimental Results and Analysis}

To order to demonstrate the effectiveness of the presented power scheduling operation, the proposed work was implemented in python programming language on a personal computer with 3.2GHz CPU and 8GB RAM. Two versions of the power scheduling algorithms are compared; one is the scheduling operation with flexible charging, and the other is without flexible charging. The data of electricity load, renewable generation, charging/discharging of EVs and battery storage facilities originated from [27],[28],[29] and [26], respectively. The power service territory of the IoE is separated into three geographical regions that mimicked three types of population distribution in the real world. The first region is an urban city with 18000 residences/buildings, and only one-tenth of them deploy renewables and battery storage facilities. This reflects the characteristics of low power supply-demand ratio as seen in a metropolitan city with high population density. 2600 residences/buildings are distributed within the geographical scope of the second region, and up to nine-tenth of the residences/buildings deploy renewables and battery storage facilities. This reflect the fact of widespread installation of renewables and battery storage facilities at most of the residences in rural areas. As for the third region, there are 2400 residences scattered in this area, and the counts of renewables and battery storage facilities are 2280. Such phenomenon with high ratio of renewable and battery storage facility deployment can be found in less cultivated or wilderness area. The numbers of private EVs, commercial EVs, and EVs for public transport are 20000, 6000, and 500, respectively [30]. As mentioned earlier, four options were offered to EV owners based on their preset preferences. The four options include slow charging station such as parking lot or workplace, fast charging station, battery swapping station, and on-road wireless charging service. The numbers of charging points that correspond with these four kinds of charging technologies are 4, 4, 2, and 2, respectively. An EV battery can be fully charged in thirty minutes for the fast charging option, whereas it may take one to six 
hours to charge an EV battery if the slow charging service is chosen [31]. The parameters of EVs and battery storage facilities used in our simulations are enumerated in Tables 1 and 2, respectively.

Table 1. Parameters of EV batteries

\begin{tabular}{|c|c|c|}
\hline Brand & BYD & Nissan \\
\hline Rated capacity (kWh) & 57 & 4.0 \\
\hline Power (kWh) & 9.5 & 15 \\
\hline Minimum SoC (\%) & 15 & 95 \\
\hline Maximum SoC (\%) & 95 & 97 \\
\hline Charging/Discharging efficiency (\%) & 97 & 14.9 \\
\hline Power consumption of the power batteries & 21.5 & 4.0 \\
\hline
\end{tabular}

Table 2. Parameters of battery storage facilities

\begin{tabular}{|c|c|c|}
\hline Type of rechargeable battery & Lead-acid & Nickel metal hydride \\
\hline Rated capacity (kWh) & 28.3 & 36 \\
\hline Power (kWh) & 5.66 & 1500 \\
\hline Cycle life (cycles) & 1000 & 4032 \\
\hline Battery operation cost (€) & 2716.8 & 10 \\
\hline Minimum SoC (\%) & 10 & 90 \\
\hline Maximum SoC (\%) & 90 & 10 \\
\hline Initial SoC (\%) & 10 & 70 \\
\hline Rated depth-of-discharge (\%) & 80 & 92.5 \\
\hline Charging/Discharging efficiency (\%) & 91.4 & \\
\hline
\end{tabular}

Fig.s 4 to 6 present the comparisons of the day-ahead power load demand, solar power, and wind power generation before scheduling for Regions 1 to 3, respectively. Notably, the EV charging load is not included in the electricity load here. As Region 1 is a highly populated metropolitan area, it can be observed from Fig. 4 that the power shortage problem becomes serious during peak load periods. This phenomenon can be interpreted by the much 
fewer renewable power systems compared with dense population in this metropolitan region. Contrarily to the results shown in Fig. 4, the solar power generated during the day time in Region 2 and the wind power systems deployed in Region 3 as shown in Figs. 5 and 6 contribute significant portion of the aggregated power supply within a day in these two regions. As a result, the supplied electricity may exceed the load demand of sparse population distribution in these two regions and the extra electricity can be stored at EV batteries and the battery storage facilities of the prosumers and microgrids, or even traded to others facing power shortage via the coordination of regional energy routers at all levels.

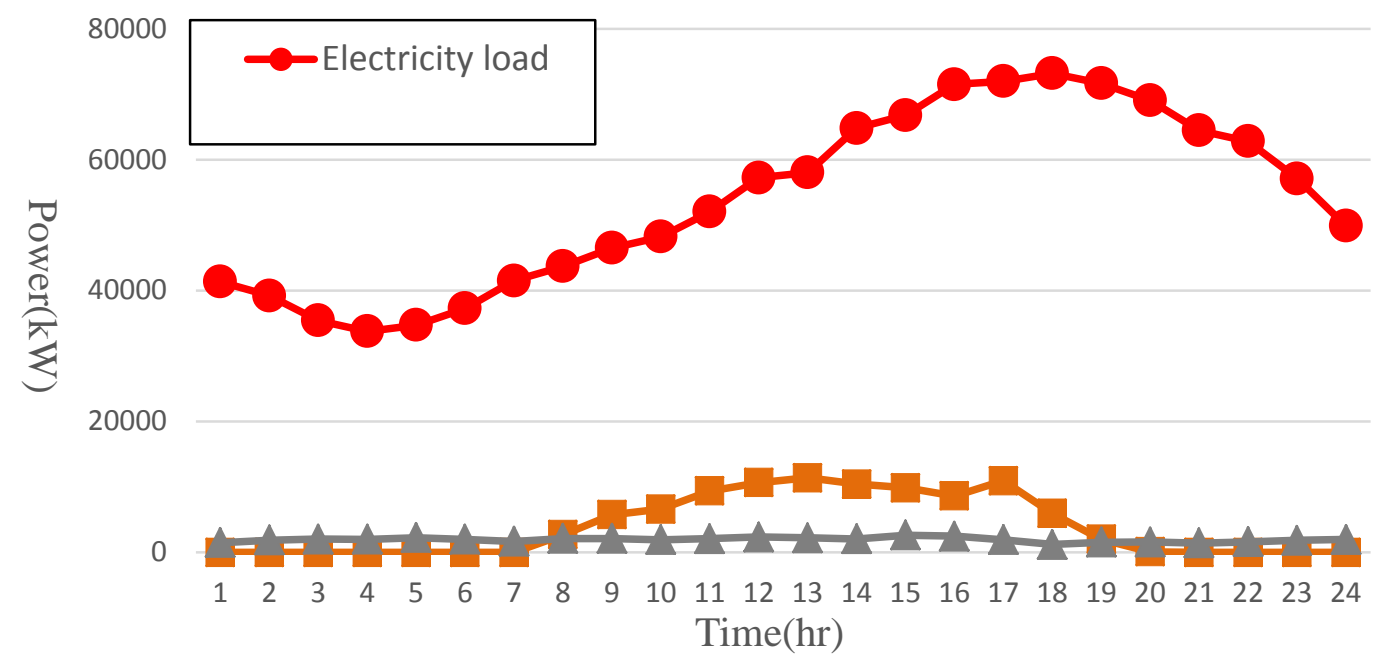

Fig. 4. Comparison of electricity load, solar power, and wind generation for Region 1 before power

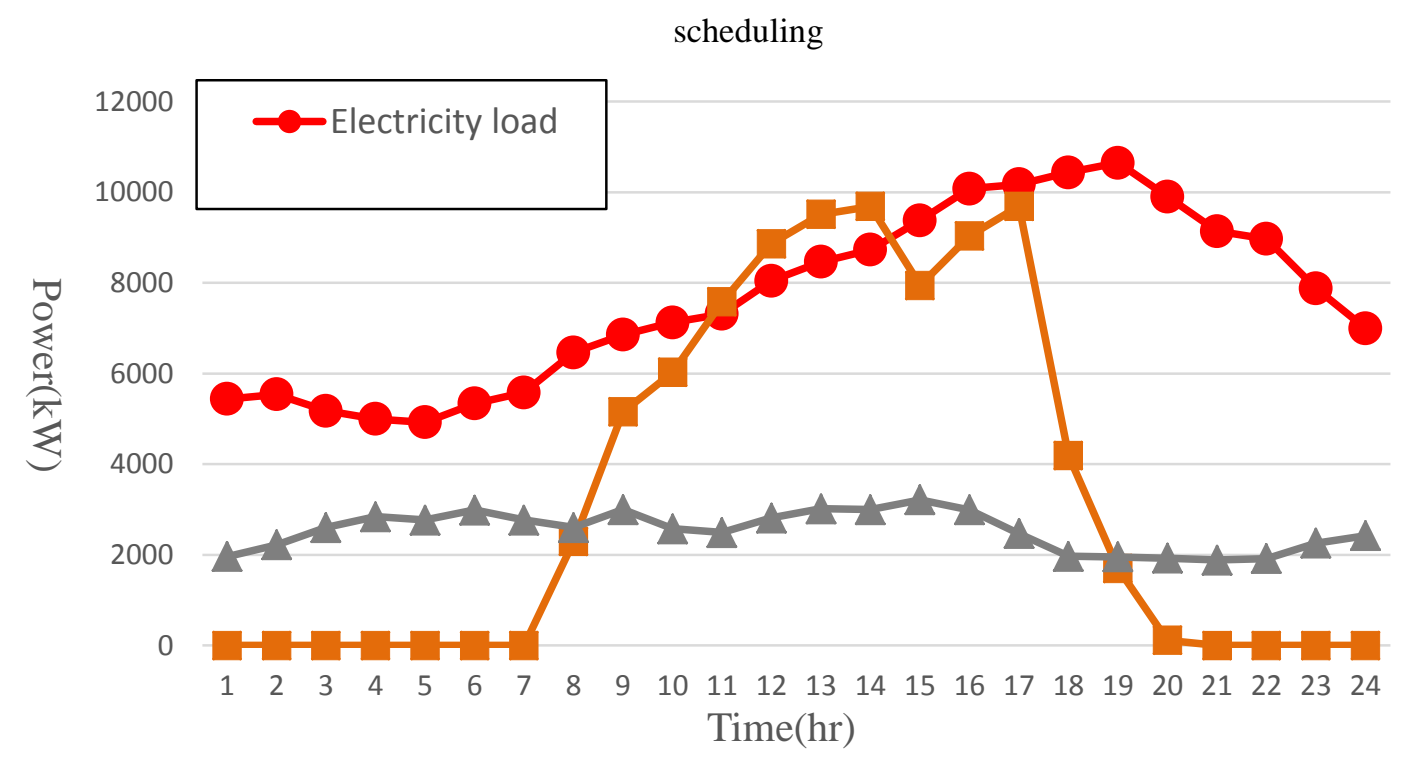

Fig. 5. Comparison of electricity load, solar power, and wind generation for Region 2 before power scheduling 


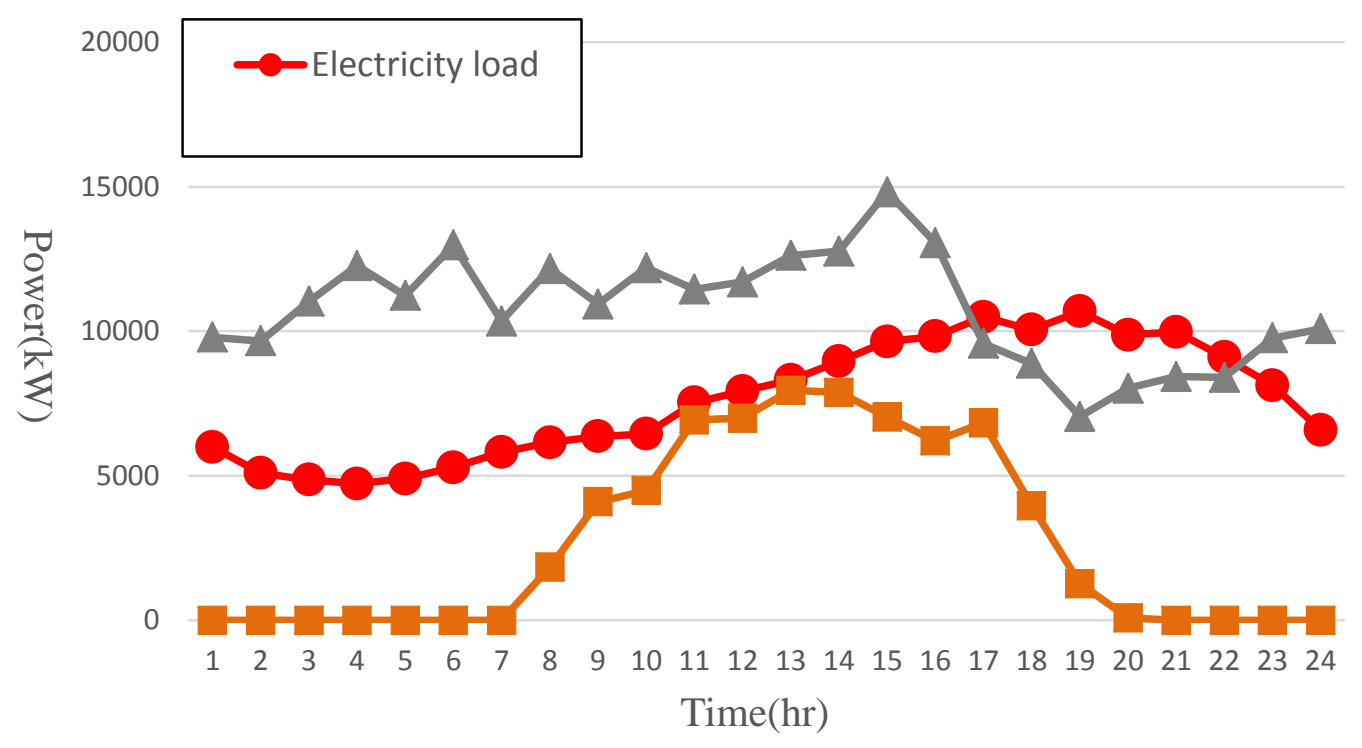

Fig. 6. Comparison of electricity load, solar power, and wind generation for Region 3 before power scheduling

Fig. 7 aggregates the electricity load, solar power generation, and wind power generation for the three regions before scheduling. Since the electricity demand in the metropolitan city is much larger than that needed in the rural and wilderness areas, it can be inferred from Fig. 7 that the power shortage occurs at the peak load periods in Region 1 results in the significant disparity between the electricity load and renewable generation for the global IoE scope during peak periods.

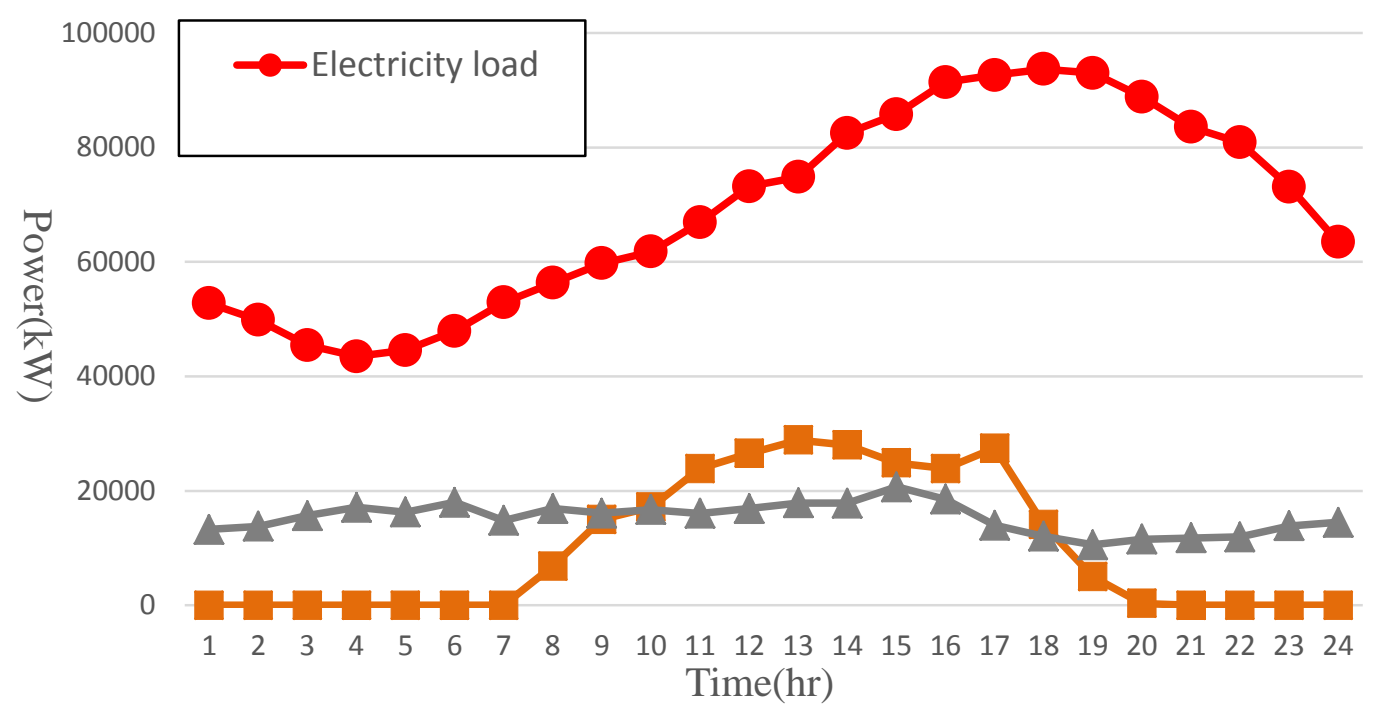

Fig. 7. Comparison of electricity load, solar power, and wind generation for the globe IoE scope before power scheduling 
The effect of flexible EV charging is illustrated in Fig. 8. The comparison target is the conventional shortest path first method. The net load in this figure is defined as the difference of the aggregated hourly electricity load and the accumulated hourly solar and wind power generation for the global IoE scope. In other words, the net load can be viewed as the subtraction of hourly renewable power generation from the corresponding electricity load as shown in Fig. 7. Consequently, positive hourly net load indicates that power scheduling or more traditional power generation is required to balance the load demand during peak load periods. It can be revealed that private EVs profoundly participate in power scheduling operation and are arranged to refill the electricity for the EV battery during off-peak periods because the private EVs are typically parked at home or the workplace most of the time. On the contrary, EVs for public transportation with fixed routes have restricted flexibility for power scheduling and charging options because they are scheduled to convey people or goods during fixed time periods of a day. As for the commercial EVs and electric taxis, they are able to shift their charging load to different charging options based on the EV owners' preferences without unbalancing the charging load of charging points. Furthermore, flexible charging approach computes the electricity demand for a moving EV required to reach the destination and restricts the EVs from charging too much power during peak load periods. The electricity load curve is thus smoothed and substantial portions of EV charging are shifted to off-peak periods.

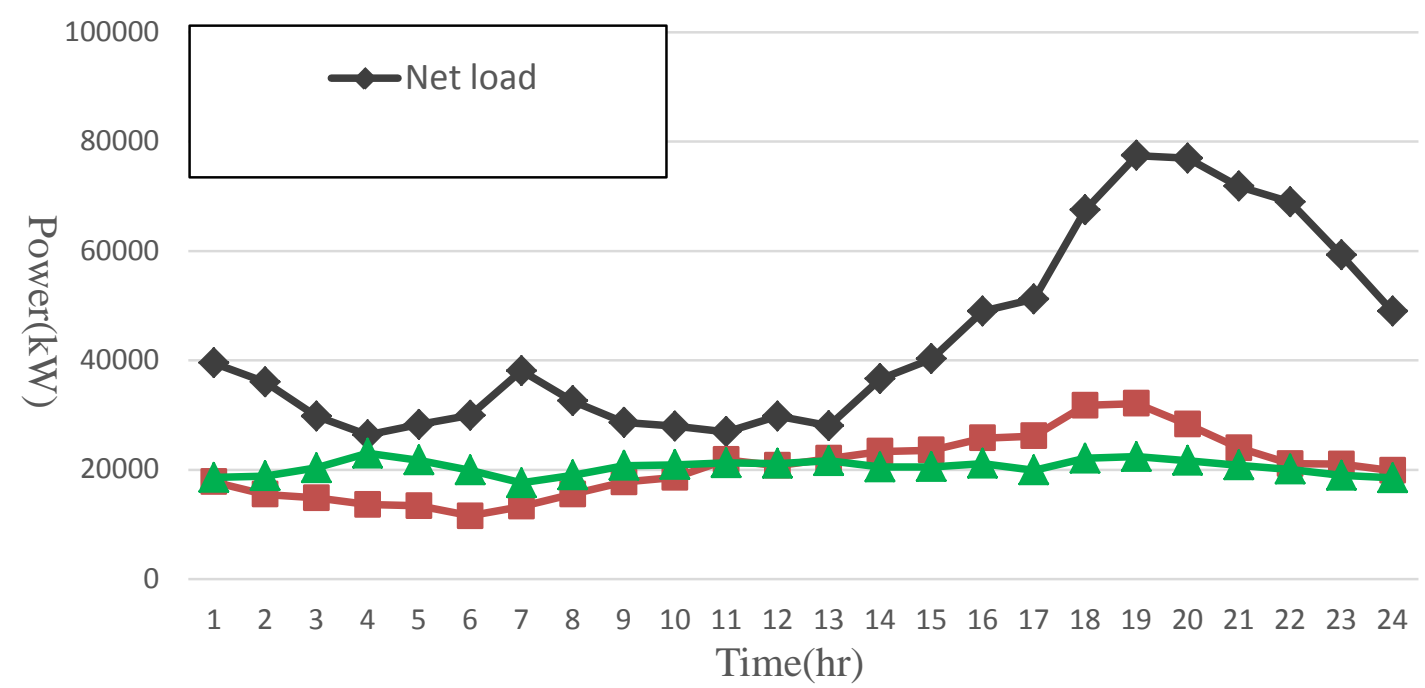

Fig. 8. Illustration of net load, EV charging load with flexible charging and shortest route approaches 
Fig. 9 shows the average time overhead required for EV battery charging with shortest route charging and flexible charging approaches. Here the time overhead is defined as the extra traveling time to the charging point plus the turnaround time spent at the charging point. As shown in Fig. 9, the flexible charging approach results in less time overhead for battery charging because the fuzzy charging point selection mechanism installed at each moving EV is informed of the congestion information at different charging points, and reduces turnaround time by selecting a less crowded charging point for the EV. Meanwhile, the proposed approach further cuts down the charging time of EVs and mitigates the peak load by controlling the amount of charging power of EVs and navigating EVs to charge the electricity during off-peak time intervals while the moving EVs park after reaching their destinations.

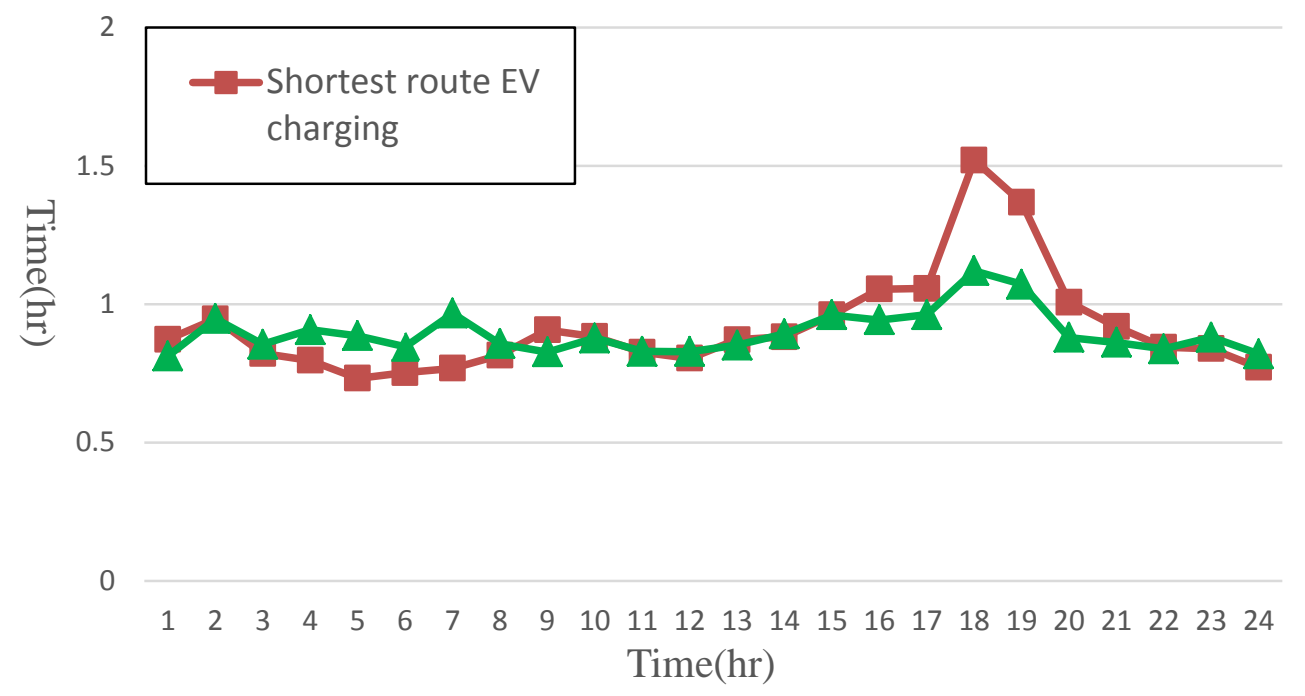

Fig. 9. Comparison of average time overhead for EVs consumed for recharging with flexible charging and shortest route approaches

The average time overhead for the $\mathrm{EV}$ groups of time-saving and cost-saving preferences is further compared in Fig. 10. A significant gap between the EVs of two groups is observed. The flexible charging approach not only balances the charging load of different charging options, but also greatly shortens the time overhead required for the EVs that request for fast charging. In addition, the flexible charging approach saves the charging cost for both EV groups at the charging points and alleviates the peak load of charging points and electricity demand by postponing portion of power recharging after a moving EV arrives at its destination. 


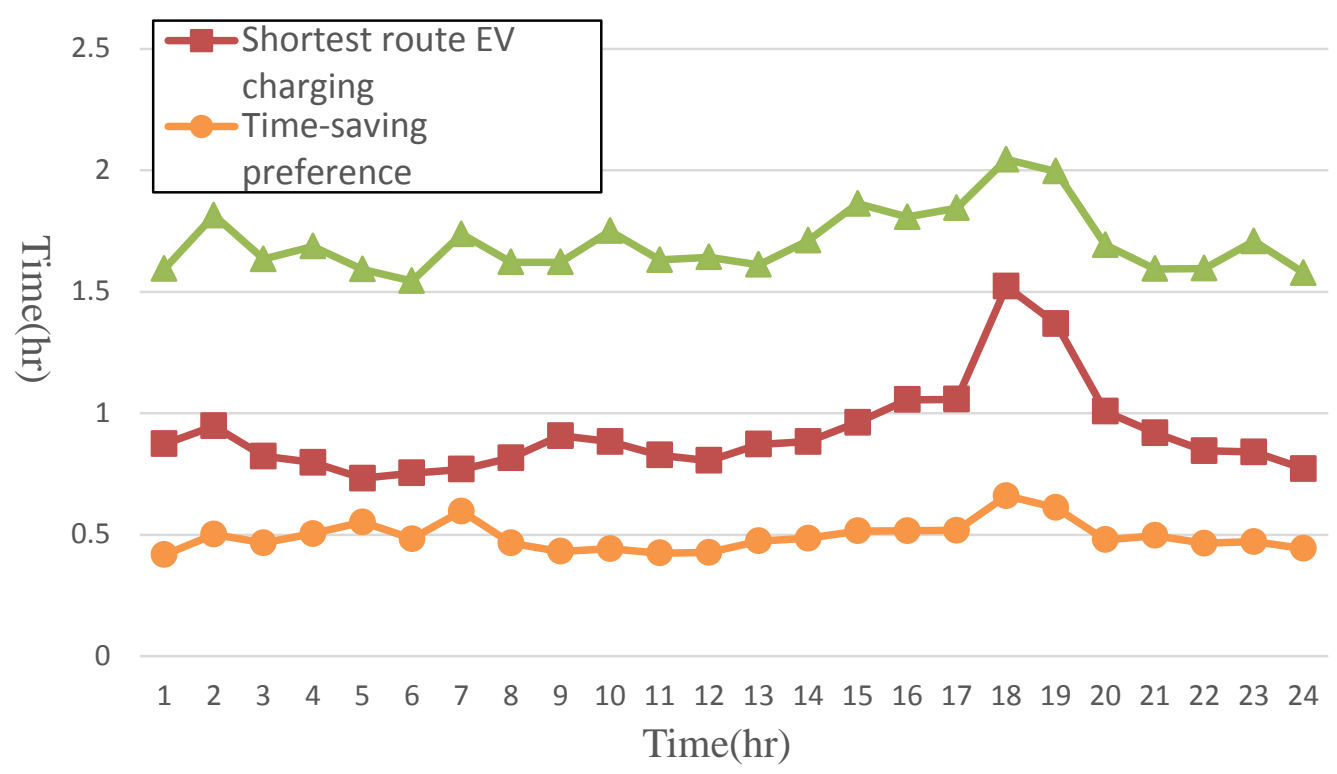

Fig. 10. Comparison of average time overhead for EV groups of time-saving and cost-saving preference

Fig. 11 illustrates the traditional power generation with flexible charging and shortest route approaches. It can be observed from the blue and red curves in this figure that the required traditional power generation is considerably declined after running the proposed power scheduling algorithm, and the flexible charging approach flattens the traditional power generation profile further. Meanwhile, portion of the surplus electricity acquired from the intermittent solar and wind electricity generation at Regions 2 and 3 are not effectively utilized because that the battery storage facilities and EV batteries within these two regions were fully charged and no available electricity sharing tool can be used to trade the surplus electricity gained from solar and wind power generation in the existing electricity management system. According to our simulation results, up to $115337 \mathrm{~kW}$ or $18.95 \%$ of the solar and wind renewable power generation are not utilized before running proposed scheduling algorithm. Contrariwise, via the trading mechanism of IoE energy router, the battery storage facilities and EV batteries at neighboring regions, such as a metropolitan area like Region 1 here, can be utilized to keep surplus electricity generated by the solar and wind power systems within the geographical territories of the rural or wilderness areas such Regions 2 and 3 in this simulation. As a result, the proposed energy router mechanism not only effectively flattens the curve of the electricity load for the global IoE scope, but also reinforces the stability of the global IoE operation. 


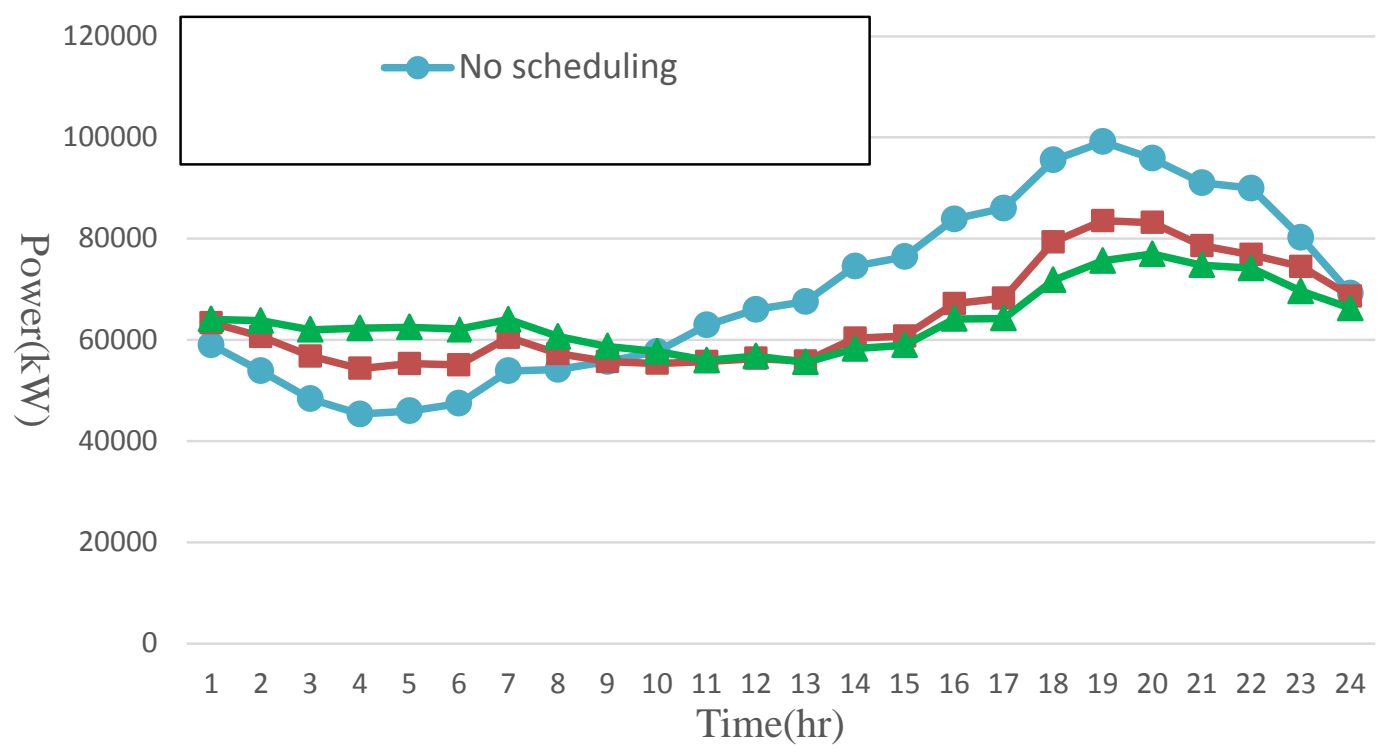

Fig. 11. Illustration of traditional power generation with flexible charging and shortest route mechanism

\section{Conclusion}

Recently, researches suggested to adopt the IoE framework to take the place of the architecture of the traditional power market. However, the uncertainty of EV movement and renewable generation, as well as the power sharing among microgrids becomes the key issues to be addressed by the operators of the power management systems for the future IoE. This work proposed a day-ahead power scheduling mechanism to address the unresolved technical issues mentioned above. After the main grid launches the day-ahead power scheduling operation, regional energy routers under a hierarchical organization are utilized to coordinate the information and power exchange among the microgrids. Notably, the regional energy routers assist in scheduling electricity load of the prosumers and EVs and allocating the surplus electricity gathered by the renewable power generation at some prosumers/microgrids to others that lack of power supply during peak load periods. The simulation results demonstrate that the proposed algorithm not only fully utilize the generated renewables to flatten the curve the load demand of the whole power market of IoE, but also ease the carbon emission problem resulting from the power generation of the traditional power plants. In addition, via the flexible charging service provided in this work, each moving EV is able to select the most suitable charging option that fits the need of each $\mathrm{EV}$ user. As a result, the charging demand for the EV users with different preferences can be 
satisfied and the congestion of charging points during peak periods were alleviated.

\section{Acknowledgements}

The authors would like to thank the Ministry of Science and Technology of the Republic of China, Taiwan for financially supporting this research under Contract Numbers MOST 106-2221-E-259-012 and MOST 107-2221-E-259-016.

\section{References}

[1] K. Zhou, S. Yang, \& Z. Shao, “Energy Internet: the business perspective,” Applied Energy, vol. 178, pp. 212-222, 2016. Article (CrossRef Link)

[2] K. Wang, J. Yu, Y. Yu, Y. Qian, D. Zeng, S. Guo, Y. Xiang, \& J. Wu, “A survey on energy internet: Architecture, approach, and emerging technologies,” IEEE systems journal, vol. 12, no. 3, pp. 2403-2416, 2018. Article (CrossRef Link)

[3] Rana, M. “Architecture of the Internet of Energy Network: An Application to Smart Grid Communications,” IEEE Access, vol. 5, pp. 4704-4710, 2017. Article (CrossRef Link)

[4] W. Hou, G. Tian, L. Guo, X. Wang, X. Zhang, \& Z. Ning, “Cooperative mechanism for energy transportation and storage in internet of energy,” IEEE Access, vol. 5, pp. 1363-1375, 2017. Article (CrossRef Link)

[5] Zhang, H., Li, Y., Gao, D. W., \& Zhou, J., "Distributed optimal energy management for energy internet,” IEEE Transactions on Industrial Informatics, vol. 13, no. 6, pp. 3081-3097, 2017. Article (CrossRef Link)

[6] Z. Zhou, F. Xiong, B. Huang, C. Xu, R. Jiao, B. Liao, Z. Yin, \& J. Li, “Game-Theoretical Energy Management for Energy Internet with Big Data-Based Renewable Power Forecasting,” IEEE Access, vol. 5, pp. 5731-5746, 2017. Article (CrossRef Link)

[7] C. C. Lin, D. J. Deng, W. Y. Liu, \& L. Chen, "Peak Load Shifting in the Internet of Energy with Energy Trading Among End-Users,” IEEE Access, vol. 5, pp. 1967-1976, 2017. Article (CrossRef Link)

[8] Du, L., Zhang, L., Tian, X., \& Lei, J., “Efficient Forecasting Scheme and Optimal Delivery Approach of Energy for the Energy Internet,” IEEE Access, vol. 6, pp. 15026-15038, 2018. $\underline{\text { Article (CrossRef Link) }}$

[9] Dou, C., Yue, D., Han, Q. L., \& Guerrero, J. M., "Multi-agent system-based event-triggered hybrid control scheme for energy Internet,” IEEE Access, vol. 5, pp. 3263-3272, 2017.

$\underline{\text { Article (CrossRef Link) }}$ 
[10] W. Tang \& Y. J. A. Zhang, “A model predictive control approach for low-complexity electric vehicle charging scheduling: optimality and scalability,” IEEE Transactions on Power Systems. Vol. 32, no. 2, pp. 1050-1063, 2017. Article (CrossRef Link)

[11] R. Wang, P. Wang, \& G. Xiao, "Two-stage mechanism for massive electric vehicle charging involving renewable energy,” IEEE Transactions on Vehicular Technology, vol. 65, no. 6, pp. 4159-4171, 2016. Article (CrossRef Link)

[12] Q. Kang, J. Wang, M. Zhou, \& A. C. Ammari, “Centralized charging strategy and scheduling algorithm for electric vehicles under a battery swapping scenario,” IEEE Transactions on Intelligent Transportation Systems, Vol. 17, no. 3, pp. 659-669, 2016. Article (CrossRef Link)

[13] A. Ghasemi, S. S. Mortazavi, \& E. Mashhour, "Hourly demand response and battery energy storage for imbalance reduction of smart distribution company embedded with electric vehicles and wind farms,” Renewable Energy, vol. 85, pp. 124-136, 2016. Article (CrossRef Link)

[14] C. C. Lin, D. J. Deng, C. C. Kuo, \& Y. L. Liang, “Optimal charging control of energy storage and electric vehicle of an individual in the internet of energy with energy trading," IEEE Transactions on Industrial Informatics, vol. 14, no. 6, pp. 2570-2578, 2018.

Article (CrossRef Link)

[15] Tan, J. \& Wang, L., "Real-time charging navigation of electric vehicles to fast charging stations: A hierarchical game approach,” IEEE Transactions on Smart Grid, vol. 8, no. 2, pp. 846-856, 2017. Article (CrossRef Link)

[16] Cao, Y., Kaiwartya, O., Wang, R., Jiang, T., Cao, Y., Aslam, N., \& Sexton, G., “Toward Efficient, Scalable, and Coordinated On-the-Move EV Charging Management,” IEEE Wireless Communications, vol. 24, no. 2, pp. 66-73, 2017. Article (CrossRef Link)

[17] Cao, Y., Yang, S., Min, G., Zhang, X., Song, H., Kaiwartya, O., \& Aslam, N., “A Cost-Efficient Communication Framework for Battery-Switch-Based Electric Vehicle Charging,” IEEE Communications Magazine, vol. 55, no. 5, pp. 162-169, 2017. Article (CrossRef Link)

[18] Zhong, W., Yu, R., Xie, S., Zhang, Y., \& Tsang, D. H., “Software Defined Networking for Flexible and Green Energy Internet,” IEEE Communications Magazine, vol. 54, no. 12, pp. 68-75, 2016. Article (CrossRef Link)

[19] Echols, A., Mukherjee, S., Mickelsen, M., \& Pantic, Z., “Communication Infrastructure for Dynamic Wireless Charging of Electric Vehicles,” in Proc. of 2017 IEEE Wireless Communications and Networking Conference, pp. 1-6, March 2017. Article (CrossRef Link)

[20] Huang, C. C., \& Lin, C. L., "Wireless power and bidirectional data transfer scheme for battery charger," IEEE Transactions on Power Electronics, vol. 33, no. 6, pp. 4679-4689, 


\section{Article (CrossRef Link)}

[21] Zhang, Y., Song, S., Shen, Z. J. M., \& Wu, C., "Robust shortest path problem with distributional uncertainty,” IEEE transactions on intelligent transportation systems, vol. 19, no. 4, 1080-1090, 2018. Article (CrossRef Link)

[22] Cao, Z., Jiang, S., Zhang, J., \& Guo, H., “A unified framework for vehicle rerouting and traffic light control to reduce traffic congestion," IEEE Transactions on Intelligent Transportation Systems, vol. 18, no. 7, pp. 1958-1973, 2017. Article (CrossRef Link)

[23] Khooban, M. H., Vafamand, N., Niknam, T., Dragicevic, T., \& Blaabjerg, F., "Model-predictive control based on Takagi-Sugeno fuzzy model for electrical vehicles delayed model,” IET Electric Power Applications, vol. 11, no. 5, pp. 918-934, 2017. Article (CrossRef Link)

[24] Ahmadian, A., Sedghi, M., \& Aliakbar-Golkar, M., "Fuzzy Load Modeling of Plug-in Electric Vehicles for Optimal Storage and DG Planning in Active Distribution Network," IEEE Transactions on Vehicular Technology, vol. 66, no. 5, pp. 3622-3631, 2017. Article (CrossRef Link)

[25] B. Zhou, X. Liu, Y. Cao, C. Li, C. Y. Chung, \& K. W. Chan, "Optimal scheduling of virtual power plant with battery degradation cost," IET Generation, Transmission \& Distribution, vol. 10 no. 3, pp. 712-725, 2016. Article (CrossRef Link)

[26] M. Mahmoodi, P. Shamsi, \& B. Fahimi, "Optimal scheduling of microgrid operation considering the time-of-use price of electricity," in Proc. of IECON IEEE 2013-39th Annual Conference of the Industrial Electronics Society, pp. 2127-2132, 2013. Article (CrossRef Link)

[27] http://www.caiso.com/TodaysOutlook/Pages/default.aspx.

[28] https://transparency.entsoe.eu/generation/r2/dayAheadGenerationForecastWindAndSolar/show.

[29] Gnann, T., Klingler, A. L., \& Kühnbach, M., "The load shift potential of plug-in electric vehicles with different amounts of charging infrastructure,” Journal of Power Sources, vol. 390, pp. 20-29, 2018. Article (CrossRef Link)

[30] Jian, L., Yongqiang, Z., \& Hyoungmi, K., "The potential and economics of EV smart charging: A case study in Shanghai. Energy Policy,” Energy policy, vol. 119, pp. 206-214, 2018.

Article (CrossRef Link)

[31] Lee, W., Schober, R., \& Wong, V. W, “An Analysis of Price Competition in Heterogeneous Electric Vehicle Charging Stations,” To appear in IEEE Transactions on Smart Grid.

Article (CrossRef Link) 


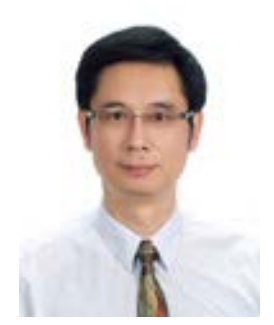

Chenn-Jung Huang received the B. S. degree in electrical engineering from National Taiwan University, Taiwan and the M. S. degree in computer science from University of Southern California, Los Angeles, in 1984 and 1987. He received the Ph. D degree in electrical engineering from National Sun Yat-Sen University, Taiwan, in 2000. He is currently a Professor in the Department of Computer Science \& Information Engineering, National Dong Hwa University, Taiwan. His research interests include computer networks, power management, e-learning, and applications of machine learning techniques.

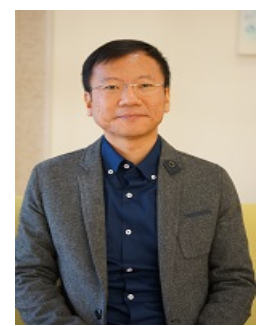

An-Feng Liu is pursuing a doctoral degree at the Department of Computer Science \& Information Engineering, National Dong Hwa University, Taiwan. Taiwan. His research interests include computer communication networks, power management, applications of machine learning techniques and e-learning.

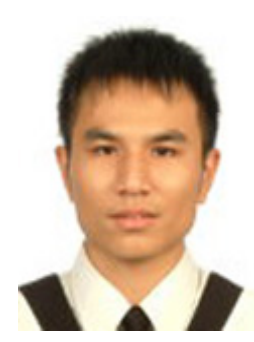

Kai-Wen Hu is pursuing a doctoral degree at the Department of Electrical Engineering, National Dong Hwa University, Taiwan. His research interests include computer communication networks, power management, applications of machine learning techniques and e-learning.

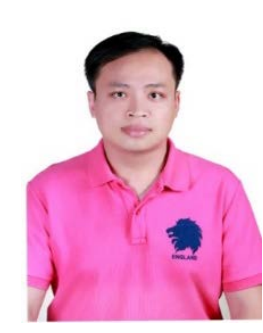

Liang-Chun Chen received the M.S. degree in institute of learning technology from the National Hualien University of Education, Hualien, Taiwan, in 2006, and the Ph.D. degree in information management from the National Taiwan University of Science and Technology, Taipei, Taiwan, in 2013. He is currently an Assistant Professor with the Department of Management, Fo Guang University, Yilan, Taiwan. His research interests include wireless protocol and power saving algorithm design in broadband access networks, social networks, and e-learning.

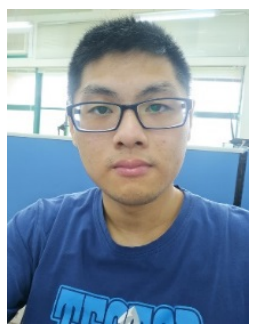

Chih-Ting Chen is pursuing a Master's degree at the Department of Computer Science and Information Engineering, National Dong Hwa University, Taiwan. His research interests include computer communication networks, data mining and applications of machine learning techniques. 